\title{
SPECT deadtime count loss correction using monitor source method
}

\author{
Wendy Siman, 2, Cheenu S Kappadath,2 \\ ${ }^{1}$ The University of Texas M. D. Anderson Cancer Center, Houston, TX, USA. \\ ${ }^{2}$ The University of Texas Graduate School of Biomedical Sciences at Houston, Houston, TX, USA.
}

Received March 19, 2014; Published Online April 08, 2014

[Presented at the Young Investigator's Symposium at the 2014 Annual Meeting of Southwest Chapter of American Association of Physicists in Medicine (AAPM) in San Antonio, Texas, USA]

\section{Conference Proceeding}

\begin{abstract}
Purpose: Deadtime-count-loss (DTloss) correction using monitor source (MS) requires: 1) uniform fractional DTloss across FOV, 2) high statistics MS images both with \& without the object. The aims are validating condition 1 and developing a practical protocol that satisfies conditions 2 with minimal additional study duration.
\end{abstract}

Methods and Materials: SPECT images of non-uniform phantoms (4GBq 99mTc) along with MS (20MBq 99mTc) attached to each detector were acquired multiple times over 48 hours in photopeak and scatter energy window (EW) using Siemens-SymbiaS and GE-D670. Planar images of the MS alone were acquired. Photopeak counts for the MS ROIs were $>100$ kcts. Fractional DTloss uniformity across the FOV was evaluated by correlating count rates in different ROIs on projection images at different DTloss levels. The correction factor for each SPECT projection at every time point was calculated as the ratio of time-corrected MS count rates with $\&$ without the phantom.

The DTloss-corrected projections for each SPECT acquisition were decay corrected to one time point. The correction accuracy was assessed against DTloss estimated by paralyzable model. The accuracy of projection-based DTloss correction for SPECT was evaluated. A method to model projection DTloss based on a subset of measured projection DTloss was investigated. The relation of DTloss between photopeak and scatter EW was explored.

Presenting author: Wendy Siman; The University of Texas M. D. Anderson Cancer Center, Houston, TX, USA.

Cite this article as:

Siman W, Kappadath SC. SPECT deadtime count loss correction using monitor source method. Int J Cancer Ther Oncol 2014;

2(2):020234. DOI: 10.14319/ijcto.0202.34
Results: The fractional DTloss was uniform across the FOV (r $>0.99$ ), validating condition 1 . The MS method was accurate to > 99\% for planar and SPECT. Measured DTloss from 3-to-5 projections/detector may be used to estimate DTloss with accuracy $>98 \%$ for all SPECT projections by modeling DTloss with measured projection rate. The correction factor in photopeak and scatter EW are equivalent with > 99\% agreement.

Conclusion: MS method can accurately correct planar and SPECT DTloss. Sparse sampling of the projection DTloss allows acquiring MS counts with high statistics with minimal additional study duration making it clinically practical. 
Monitor Source Method to compensate for deadtime loss

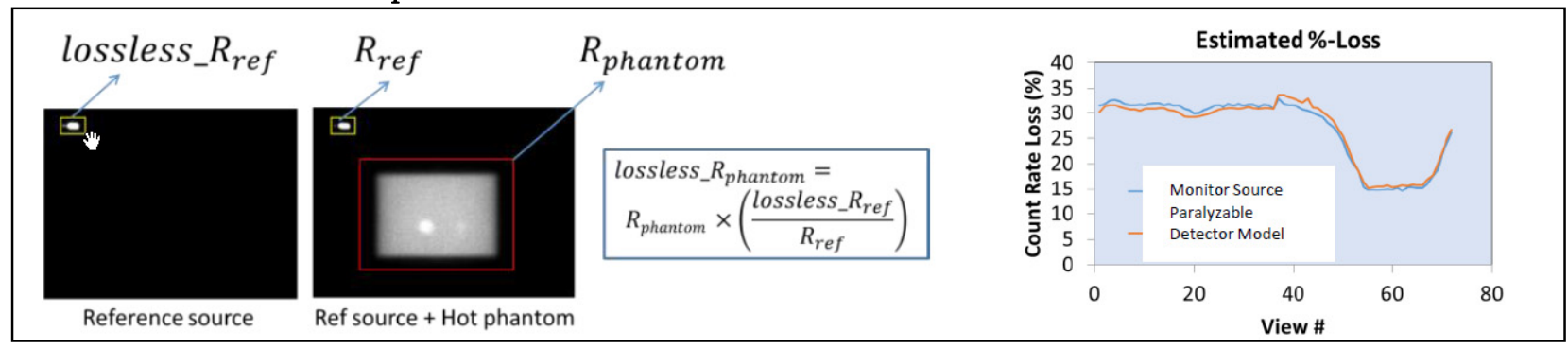

Images of monitor source $\mathrm{w} /$ and w/o phantom to calculate deadtime loss \& equivalence with paralyzable detector model. Special considerations: (1) Correction accuracy depends on monitor source counting statistic; (2) Shield monitor source ROI from external signal.

\section{Relative count loss uniformity in FOV}
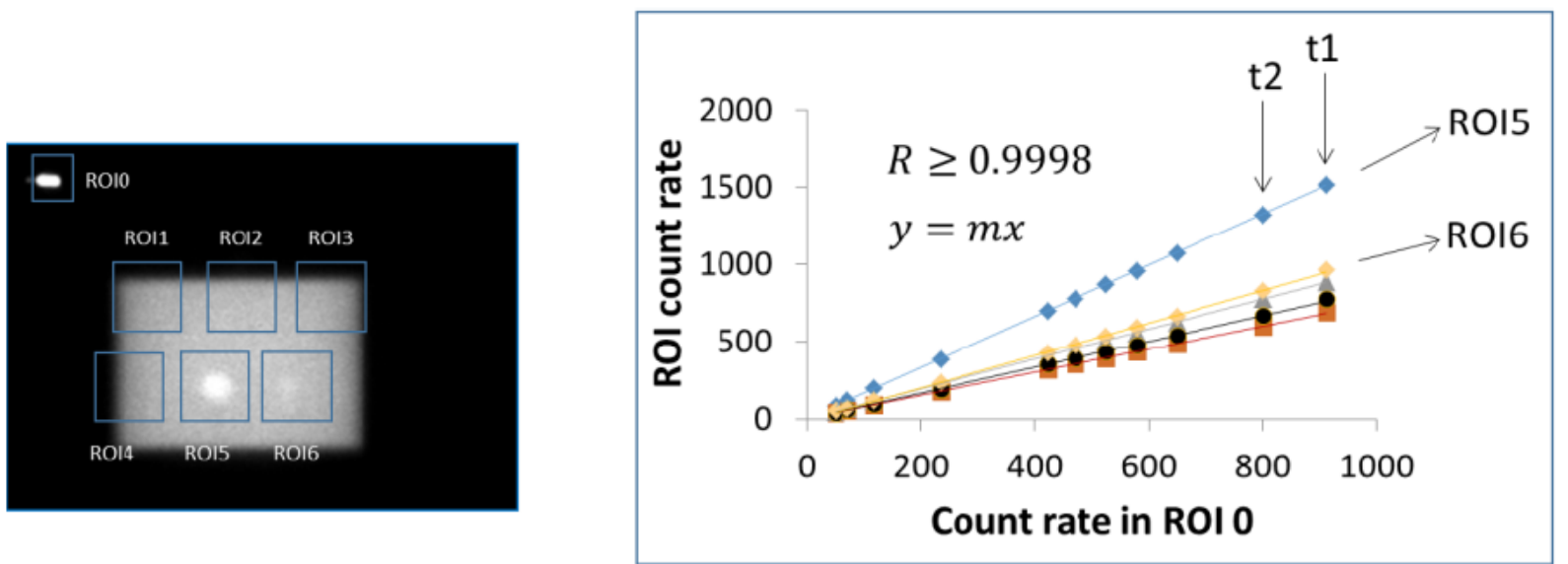

Each ROI count rates have fix proportionality at all count loss as source decays, i.e., they all have the same fractional count loss; hence a frame based correction and monitor source method is valid. Results hold true under different phantom configurations.

Accuracy of deadtime corrections (same for photopeak\& scatter)

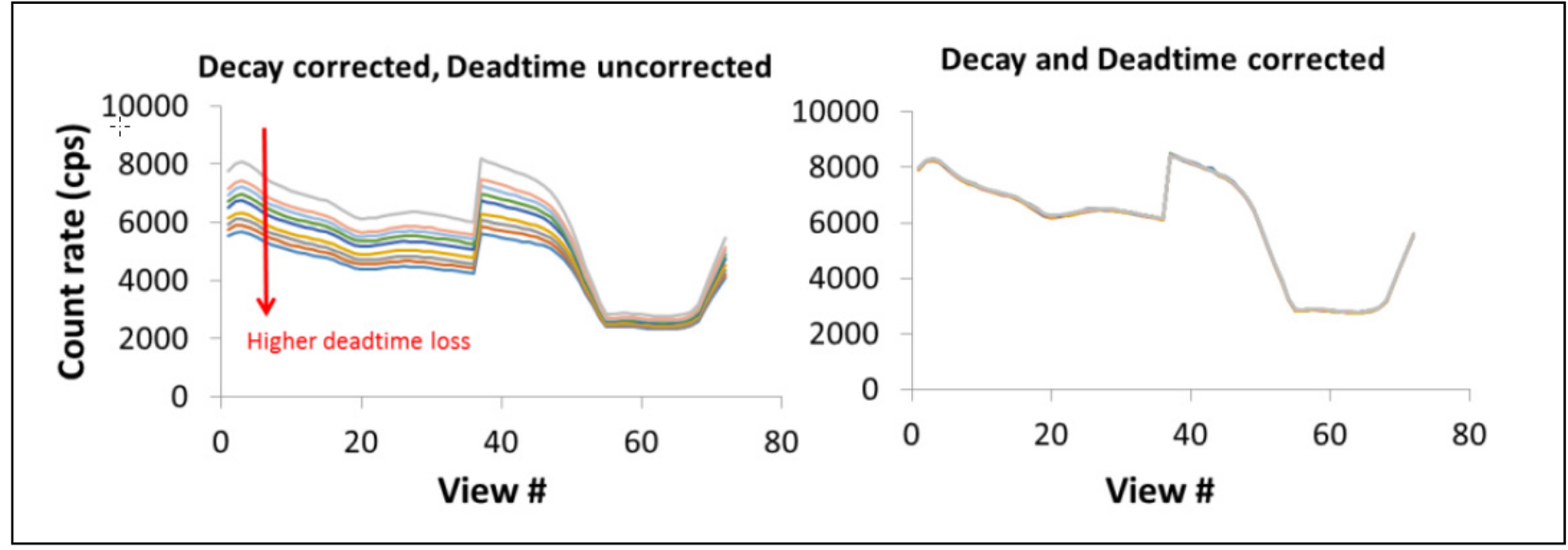

SPECT projection rates with losses. After both deadtime loss and decay correction to a single time point, all projection count rates agree within $<1 \%$ with maximum $\mathrm{COV}<0.8 \%$. 
Sparse sampling: monitor source method is performed in 3 or 5 projections only

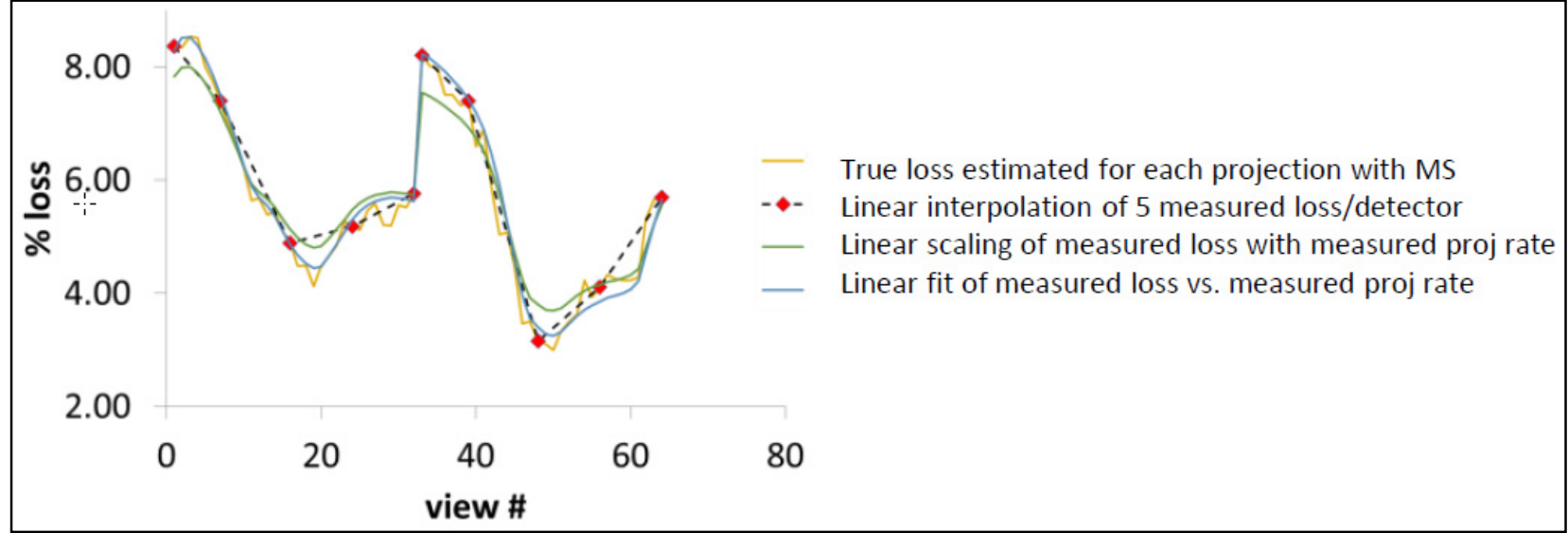

The true losses for all projections compared against those modeled from losses measured for 5 proj/det. Three different modeling/interpolation methods were found to have accuracy $>98 \%$. Losses for minimum of 3 proj/det required to interpolate losses in all other projections. 